\title{
The Effect of Competition on Salivary Testosterone in Elite Female Athletes
}

\author{
Lisa Dawn Hamilton, Sari M. van Anders, David N. Cox, \\ and Neil V. Watson
}

The association between androgens and competition in women has been understudied compared with men. The current study examined the link between testosterone (T) and competition in elite female athletes, using a sample of female wrestlers that included athletes competing at both the national and international level. In a repeated-measures design, saliva samples were collected before and after wrestling bouts, with comparable samples of wins and losses, and subsequently analyzed for T. Study results showed a $22 \%$ increase in circulating bioavailable $\mathrm{T}$ from preto postbout, $F(1,12)=9.71, P=.009$. There was no significant difference in T between win or loss outcomes. These findings—-showing a link between individual head-to-head competition and T in women - demonstrate that women's androgenic responses to environmental contexts are dynamic and may be an important factor to address in research on competitive performance.

Keywords: testosterone, androgen, competition, challenge hypothesis, women, sports

Androgens — especially testosterone ( T) — and competitive behaviors appear to modulate each other reciprocally. The dynamic nature of the testosterone-competition relationship has been well studied in men, but is poorly understood in women (for a review, see ${ }^{1}$ ). Physiologically, T has similar effects in men and women, such as probable importance for aspects of competition ranging from muscle development to alterations of neurocognitive processing.

Previous studies of women's T responses to competition have produced mixed results. Female rugby ${ }^{2}$ and soccer players ${ }^{3}$ showed significant increases in salivary $\mathrm{T}$ in response to regular season matches. However, other studies have failed to find a significant change in women's androgen levels in response to competition (eg, handball or volleyball, ${ }^{4}$ and rowing $^{5}$ ).

Given that $\mathrm{T}$ response to competition has been observed in women mainly in college team sports, the current study was designed to sharpen the focus on athletic performance by sampling elite-level athletes in an individual sport. Because Simon Fraser University houses a Canadian national training center for wrestling, we were able to test a sample of nationally and internationally ranked female wrestlers. Wrestling also was chosen because none of the previously published reports have looked at individual head-to-head competition in women.

The authors are with the Department of Psychology, Simon Fraser University, Burnaby, BC, Canada. 
We hypothesized that $\mathrm{T}$ would increase in response to competition. We were also interested in examining whether $\mathrm{T}$ would change as a function of the competition outcome, with higher $\mathrm{T}$ following a win in comparison with a loss, as has been seen in male wrestlers. ${ }^{6}$

\section{Methods}

\section{Participants}

The participants were 21 female wrestlers, and most were nationally ranked. Within this sample were athletes who have competed in the Olympics or World Championships, including individuals who had medaled at these events. Of these participants, 13 had saliva samples for both a win and a loss, and the demographic characteristics of these participants are reported in Table 1 . Five of the 13 participants reported using hormonal contraceptives; although they modulate T in normal circulation, contraceptives reportedly do not attenuate competition-mediated increases in T. ${ }^{7}$ The study employed a within-subjects design, so participants served as their own controls and any differences in baseline $\mathrm{T}$ or any other individual differences would be present in both win and loss conditions. All procedures were approved by the Simon Fraser University Research Ethics Board, and all participants provided written informed consent.

\section{Measures}

Demographics Questionnaire. This questionnaire covered basic demographic information for the participants, as well as specific health information required for this study.

Testosterone. Participants collected their own saliva by chewing sugarless gum and salivating into polystyrene test tubes. Saliva was stored at $-20^{\circ} \mathrm{C}$ until

Table 1 Participants' demographic information

\begin{tabular}{lcc}
\hline & \multicolumn{2}{c}{ Participants $\mathbf{n}=\mathbf{1 3})$} \\
\cline { 2 - 3 } & $\mathbf{M}$ & $\mathbf{S D}$ \\
\hline Age & 20.5 & 1.8 \\
& $\mathbf{n}$ & $\%$ \\
\cline { 2 - 3 } Education & & \\
College Student & 12 & 92.5 \\
College Graduate & 1 & 7.5 \\
Ethnicity & & 85 \\
$\quad$ Caucasian & 11 & 7.5 \\
Canadian & 1 & 7.5 \\
$\quad$ Mixed Ethnicity & 1 & 38 \\
Hormonal Contraception & & 3 \\
$\quad$ Currently Using & 5 & \\
\hline
\end{tabular}


processing. Salivary testosterone radioimmunoassays were performed in duplicate by the Biomarkers Core Laboratory at the Yerkes Primate Research Center, Emory University, with interassay CV of $8.7 \%$ and intraassay CV of 6.5\%. Samples were always collected in the afternoon and early evening.

\section{Procedures}

The study had a repeated-measures design; whenever possible, we collected saliva from each participant both before and after a win and before and after a loss. Competitions took place during scrimmages and tournaments. It is important to note that because these elite wrestlers are continually in competition for national ranking, their levels of motivation are consistently high for both types of bouts. Data were collected over two tournaments and four scrimmages. Twenty minutes before competitions, participants provided a precompetition saliva sample. Approximately 10 min after each competition of interest, the participants provided a second, postcompetition saliva sample. Samples were gathered from participants on subsequent days until a postwin and a postloss sample had been obtained from each participant.

\section{Data Analysis}

We used data from participants who had both a win and a loss in either a tournament or a scrimmage $(n=13)$. Five of the 13 participants had wins and losses in both conditions, in which case we randomly chose to use either the scrimmage or tournament data. Analyses were run as $2 \times 2$ repeated-measures ANOVAs testing the effect of time (pre- and post-) $\times$ outcome (win and loss) on T.

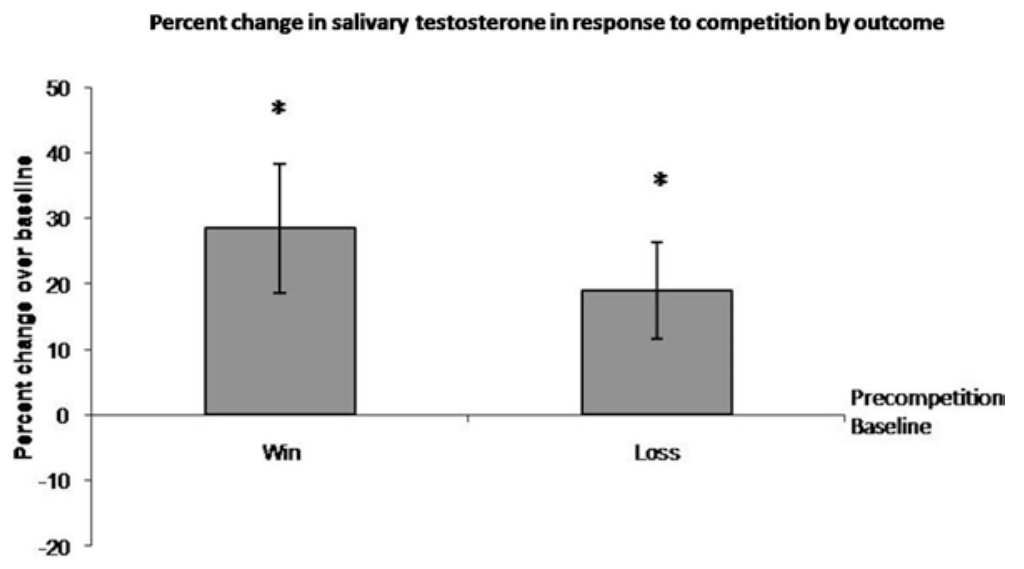

Outcome

Figure 1 - Percent change over baseline (prebout to postbout) for salivary T $(n=13)$ for wins and losses $( \pm$ SEM). Testosterone increased significantly over baseline regardless of the competition outcome. *Indicates a significant difference from zero at $p<.05$. 


\section{Results}

The repeated-measures ANOVA revealed a significant effect of time, $F(1,12)=$ $9.71, P=.009$, such that $\mathrm{T}$ increased from an average prematch value of $30.1 \mathrm{pg} /$ $\mathrm{mL}(S D=10.5)$ to $35.2 \mathrm{pg} / \mathrm{mL}(S D=11.9)$ postmatch. On average, participants' $\mathrm{T}$ increased by $22 \%$ (95\% C.I. $=9$ to $36 \%)$. There was no significant effect of win-loss outcome, $F(1,12)=.48, P=.50$ and no significant interaction between time and outcome, $F(1,12)=.16, p=.70$ (Figure 1$)$.

\section{Discussion}

The current study examined the effects of individual competition on salivary $\mathrm{T}$ levels in elite female wrestlers. We found that the athletes' testosterone levels increased in response to intense competition, regardless of outcome. Our data are the first to show increased $\mathrm{T}$ following individual athletic competitions in women. Testosterone decreases over the day, and previous data from our laboratory shows decreases in women's $\mathrm{T}$ during the time frame employed in this study. ${ }^{8}$ As such, increased T over competitions could not be attributed merely to the passage of time.

We did not observe any significant difference in the induced change in testosterone levels when comparisons were made between wins and losses, as has been demonstrated in men's competition. ${ }^{1}$ The absence of any difference in T response between wins and losses in this sample of women wrestlers adds support to the growing body of data indicating that a significant outcome-related change in testosterone does not occur in women. ${ }^{2,3}$ However, it remains possible that in women there is a weaker competition effect that has yet to be effectively demonstrated.

\section{Practical Implications}

The observation that women athletes show an androgenic response to intense head-to-head competition raises the question of the functional significance of the induced changes. It is possible that increased testosterone can enhance performance through alteration of cognitive or physical processes.

\section{Conclusions}

Our results are in good agreement with the challenge hypothesis, ${ }^{9}$ which posits that in many species, individual competition precipitates a rise in testosterone as part of a coordinated response to challenge.

\section{Acknowledgments}

The authors are grateful to the athletes and coaches whose participation made this study possible. Funded by Natural Sciences and Engineering Research Council of Canada operating grant 0194522 to NVW. 


\section{References}

1. Van Anders SM, Watson NV. Social neuroendocrinology: Effects of social contexts and behaviours on sex steroids in humans. Hum Nat. 2006;17:212-237.

2. Bateup HS, Booth A, Shirtcliff EA, Granger DA. Testosterone, cortisol, and women's competition. Evol Hum Behav. 2002;23:181-192.

3. Edwards DA, Wetzel K, Wyner DR. Intercollegiate soccer: Saliva cortisol and testosterone are elevated during competition, and testosterone is related to status and social connectedness with teammates. Physiol Behav. 2006;87:135-143.

4. Filaire E, Le Scanff C, Duché P, Lac G. The relationship between salivary adrenocortical hormones changes and personality in elite female athletes during handball and volleyball competition. Res Q Exerc Sport. 1999;70:297-302.

5. Kivlighan KT, Granger DA, Booth A. Gender differences in testosterone and cortisol response to competition. Psychoneuroendocrinology. 2005;30:58-71.

6. Elias M. Serum cortisol, testosterone, and testosterone binding globulin responses to competitive fighting in human males. Aggress Behav. 1981;7:215-222.

7. Edwards DA, O'Neal JL. Oral contraceptives decrease saliva testosterone but do not affect the rise in testosterone associated with athletic competition. Horm Behav., in press.

8. Van Anders SM, Watson NV. Ability- vs. chance-determined competition outcomes: Effects on testosterone in humans. Physiol Behav. 2007;90:634-642.

9. Wingfield JC, Hegner RE, Dufty AM, Ball GF. The 'Challenge Hypothesis': Theoretical implications for patterns of testosterone secretion, mating systems and breeding strategies. Am Nat. 1990;136:829-846. 